\title{
SAÚDE BUCAL NA ESTRATÉGIA SAÚDE DA FAMÍLIA NO MUNICÍPIO DE SÃO PAULO: PERSPECTIVA DO USUÁRIO
}

\author{
BUCCAL HEALTH IN FAMILY HEALTH STRATEGY IN THE \\ CITY OF SÃO PAULO: THE PATIENT'S PERCEPTIONS
}

Mara Silvia Galletti Munkeviz

Maria Cecilia Focesi Pelicioni ${ }^{2}$

Munkevis MSG; Pelicioni MCF. Saúde Bucal na Estratégia Saúde da Família no município de São Paulo: uma perspectiva do usuário. Rev. Bras. Cresc. e Desenv. Hum. 2010, 20(3) 791-801

\section{Resumo:}

O presente trabalho trata da recente inserção da saúde bucal no Programa Saúde da Família (PSF) identificando as opiniões, percepções e necessidades dos usuários adultos da UBS Vila Espanhola, município de São Paulo. Para sua realização foi feita uma pesquisa qualitativa, composta pelas seguintes etapas: observação sistemática das atividades da equipe de saúde bucal da UBS e entrevista com os usuários adultos, maiores de 18 anos, que tiveram algum tipo de atendimento odontológico na UBS nos últimos 6 meses. Na fase de análise optou-se pela técnica de análise de conteúdo de Bardin. Percebeu-se que a inserção do PSF aumentou o acesso da população estudada na região da UBS, o que trouxe maior demanda por serviços especializados em odontologia. Contudo, os usuários não estavam conseguindo utilizar esses serviços de atenção secundária em saúde bucal. Algumas insatisfações foram detectadas: a constante troca de dentistas na equipe, comprometendo o vínculo, assim como os critérios e prioridades nos atendimentos. A inserção da saúde bucal no PSF trouxe alguns avanços, porém precisa ser constantemente reavaliada para realmente conseguir atingir suas principais propostas, como a integralidade da atenção, a equidade, entre outras.

Palavras-chave: saúde bucal coletiva; Programa Saúde da Família; percepção do usuário.

Departamento de Prática de Saúde - Faculdade de Saúde Pública - Universidade de São Paulo - Avenida Dr. Arnaldo, 715 CEP: 01246-904 São Paulo - SP. E-mail para contato: ceciliafocesipelicioni@yahoo.com.br

1 Mestre em Saúde Pública pela Faculdade de Saúde Pública/ USP

2 Professora doutora, livre docente da Faculdade de Saúde Pública/USP

O artigo tem como base a Dissertação de Mestrado intitulada: Saúde Bucal no Programa Saúde da Família no município de São Paulo: uma perspectiva do usuário. Apresentada em 2009 para a Faculdade de Saúde Pública da Universidade de São Paulo. 


\begin{abstract}
This work is about the insertion of oral health in the Family Health Program - PSF identifying opinions, perceptions and necessities of the adult's patients from Vila Espanhola UBS (Health Basic Unity), São Paulo city, in relation to dental health and the service of attention to buccal health offered by the PSF. The methodology was qualitative research made of the following steps: systematic observation of the activities done by the buccal health team of the UBS, and interview with the adult patients, older than 18 , that had any kind of attention in the past six months. In the analyses phase, we opted by the content analysis of Bardin. It was noticed that the insertion of PSF increased the access of the studied population in the UBS region, which increase brought a bigger demand by specialized oral services. The patients are not being able to have access to those secondary attentions in buccal health. Some dissatisfaction were detected: the constant changing of the dentists in the team compromising the link, lack of patients' autonomy in relation to their own oral health, as the way how it is applied the equity principle to prioritize the cares. Then, the insertion of buccal health in the PSF brought some advances, however it needs to be reevaluated to really reach its main proposals as integrality of attention, equity among others needs.
\end{abstract}

Key word: collective buccal health; family health program; patient's perceptions.

\section{INTRODUÇÃO}

A Saúde da Família entendida como estratégia de reorientação do modelo assistencial teve a inserção da odontologia a partir de 2000, o que foi considerada uma verdadeira revolução no cenário da saúde bucal coletiva sob a conjuntura de uma política pioneira no sentido de não somente, buscar melhorias no atendimento, mas ainda, de atingir maior inclusão social.

De acordo com o Ministério da Saúde ${ }^{1}$, o objetivo geral dessa inserção no PSF foi ampliar o acesso da população às ações de saúde bucal, incluindo-as no conjunto de atividades desenvolvidas pelo PSF, respeitando seus princípios organizativos e operacionais, estimulando dessa forma a reorganização da saúde bucal na atenção básica.

Antes da inclusão da saúde bucal no PSF, $\mathrm{o}$ atendimento odontológico brasileiro esteve durante longo período voltado, quase que ex- clusivamente, às crianças de 7 a 12 anos. No entanto, esse enfoque excluiu quase que totalmente as demais faixas etárias da assistência à saúde bucal. Constata-se que, em relação aos índices de cárie dentária, os progressos experimentados pela faixa etária focada, ou seja, de 7 a 12 anos, estão muito distantes dos índices dos jovens e adultos. Estudo de 2002, realizado no Estado de São Paulo mostra uma prevalência persistentemente alta de cárie nos adultos ${ }^{1}$.

Segundo o $\mathrm{IBGE}^{2}$, até o ano de 2003, quase 28 milhões de brasileiros nunca haviam ido ao dentista e durante muito tempo os serviços públicos no Brasil e as entidades com eles conveniadas tiveram o predomínio do paradigma cirúrgico-restaurador, pelo qual nas crianças era dada ênfase ao tratamento restaurador e nos adultos a situação era usualmente pior, pois, em um expressivo número de serviços, as extrações em massa se constituíam na única forma de tratamento disponível. 
Portanto, a inserção da saúde bucal no Programa Saúde da Família (PSF) pode representar um avanço nessa dívida que o exercício da saúde bucal brasileira tem com os mesmos, principalmente levando-se em conta os adultos.

O PSF veio trazer uma nova forma de atuação que tem como norma focar a família como seu principal eixo de trabalho consolidando um novo modelo de atendimento em saúde bucal que vai romper com os modelos de assistência vigentes, que até aqui se caracterizaram ora como curativos-mutiladores, ora como promotores de saúde, mas apenas para a população infantil escolar.

Apesar de ocorrer a municipalização, o PSF foi implantado no município da mesma forma como estava sendo trabalhado com o Projeto QUALIS - Qualidade Integral em Saúde, com instituições parceiras, constituindo uma rede de apoio, pois a Secretaria Municipal de Saúde entendia que não era possível assumir tal compromisso sozinha. Assim, foi institucionalizada a Rede de Apoio mediante convênios, nos quais instituições de ensino e serviço pactuaram uma agenda de trabalho, tendo em vista a capacitação e formação de profissionais aptos a construir o PSF nas (os) diferentes regiões/distritos da cidade.

A inclusão da saúde bucal no PSF, no Brasil e principalmente em uma cidade grande como São Paulo, enfrenta muitos problemas e desperta um interessante debate, pois permite vislumbrar o nascimento de outra saúde bucal, essa sim resolutiva e integral e oferecendo maior acesso aos usuários.

A saúde bucal, que sempre caminhou às margens do sistema de saúde, tendo uma atenção mais voltada à criança, com a grande parte da oferta dos programas dirigidos ao segmento infantil, tem agora no PSF uma melhor perspectiva de atuação: "a nova saúde bucal que se espera surgir no interior da ESF não deve guardar lugar para ações e serviços que privilegiam faixas etárias ou grupos específicos, cujas ações 'inovadoras' não vão além das monóto- nas palestras de 'educação' para a saúde que buscam 'ensinar' aos pobres como cuidar de suas maltratadas bocas. Não há mais espaço para o atendimento da maioria da população em filas intermináveis e desumanas"

Assim, os objetivos são de identificar as opiniões, percepções e necessidades dos usuários adultos da Unidade Básica de Saúde Vila Espanhola, SP em relação à sua saúde bucal e ao PSF.

\section{MÉTODO}

O cenário de estudo da pesquisa foi a Unidade Básica de Saúde Vila Espanhola que está localizada no bairro da Vila Nova Cachoeirinha, região norte do município de São Paulo. A opção por essa Unidade se deu por ser uma das que trabalha há mais tempo com saúde bucal dentro do PSF no município de São Paulo.

A população de estudo foi constituída por usuários adultos maiores de 18 anos, que não possuíam nenhuma incapacidade mental que pudesse comprometer a compreensão da pesquisa e que utilizavam o PSF para a saúde bucal naquela UBS.

Esses usuários deveriam ter tido algum tipo de atendimento pela equipe de saúde bucal nos últimos seis meses, para poderem dar suas opiniões sobre o programa.

A escolha dessa população foi não probabilística e intencional, o número de sujeitos entrevistados foi definido de acordo com critérios de saturação.

Utilizou-se a metodologia qualitativa. Sabe-se que em pesquisa qualitativa não importa quantos sujeitos serão entrevistados, pois isso tem importância relativamente secundária em relação à questão "quem?". "O que há de mais significativo nas amostras intencionais ou propositais não se encontra na quantidade final de seus elementos $(0$ "N" dos epidemiologistas), mas na maneira como 
se concebe a representatividade desses elementos e na qualidade das informações obtidas deles" 4 .

Como instrumentos para a coleta de dados optou-se pela observação sistemática do serviço, a pesquisa documental e as entrevistas com os usuários realizadas por meio de formulário. O roteiro de entrevista foi semiestruturado, combinando questões fechadas e abertas.

A observação sistemática foi a primeira etapa da pesquisa de campo. A observação como técnica de coleta de dados foi feita para descrever o tipo de serviço que é realizado na Unidade Básica de Saúde Vila Espanhola, visto que o pesquisador não conhecia a dinâmica do serviço e achou mais adequado conhecer o funcionamento das atividades das equipes de saúde bucal antes de iniciar as entrevistas com os usuários.

Durante os meses de março, abril e maio de 2008, em dois dias da semana, foi feito um acompanhamento das atividades das equipes de saúde bucal incluindo o atendimento aos usuários, as visitas domiciliares e os trabalhos dirigidos aos grupos, entre outros.

As observações foram registradas em um diário de campo. A observação tornou-se estimulante na medida em que os profissionais foram deixando o pesquisador mais à vontade considerando-o como parte do grupo.

A partir daí, também teve-se acesso a alguns documentos na Unidade Básica de Saúde: as agendas de atendimentos, as fichas clínicas,os prontuários de atendimentos e cartões-família. Assim, concomitantemente à observação sistemática fazia-se a pesquisa documental, cujas informações foram sendo coletadas para posterior análise. As informações documentais são relevantes para estudos de caso, e sua utilidade maior é a de "corroborar e valorizar as evidências oriundas de outras fontes" 5 .

$\mathrm{Na}$ pesquisa documental foi feito um levantamento das informações consideradas relevantes sobre a produção mensal das Equipes de Saúde Bucal. Verificou-se o número de usuários que iniciavam tratamento, o número de tratamentos completados, quantos eram atendidos em urgência e quantos procedimentos coletivos de prevenção eram feitos por mês.

Estas informações coletadas puderam ser relacionadas e comparadas com os dados qualitativos das entrevistas feitas posteriormente a fim de fazer uma triangulação: "a análise dos dados levantados através deste desenho metodológico (a triangulação) consiste na comparação entre os diferentes discursos levantados e os dados quantitativos obtidos através de consulta a fontes de dados secundários" ${ }^{6}$.

E enfim, foram feitas as entrevistas, realizadas pela própria pesquisadora no mês de junho de 2008. Com o apoio da equipe de saúde bucal e com base nos formulários de atendimento foram selecionados alguns usuários de acordo com o critério de inclusão determinado na metodologia da pesquisa. Conforme a disponibilidade e a opção do usuário de participar ou não da pesquisa foram agendadas as entrevistas na própria UBS Vila Espanhola em dia e horário adequado ao usuário.

"O que torna a entrevista instrumento privilegiado de coleta de informações para as ciências sociais é a possibilidade de a fala ser reveladora de condições estruturais, de sistemas de valores, normas e símbolos (sendo ela mesma um deles) e ao mesmo tempo ter a magia de transmitir, através de um porta voz, as representações de grupos determinados, em condições históricas, socioeconômicas e culturais específicas" 7 .

Para a análise dos dados coletados, decidiu-se pela utilização do método de Bardin, a análise de conteúdo, que, segundo a autora, "trata de um conjunto de técnicas de análise de comunicação, visando a obter, por procedimentos sistemáticos e objetivos de descrição do conteúdo das mensagens, indicadores que permitem a inferência de conhecimentos relativos às condições de produção/ recepção destas mensagens" 8 . 
Foram selecionados fragmentos das falas obtidas que expressavam as idéias dos sujeitos entrevistados levando à discussão de alguns assuntos. Tais assuntos deram origem a quatro temas fundamentais: o vínculo entre usuário e profissional de saúde, o acesso aos serviços de saúde bucal, a integralidade da atenção em saúde bucal e a equidade no trabalho da equipe.

\section{RESULTADOS E DISCUSSÃO}

Por meio da observação sistemática pode-se conhecer como os usuários eram atendidos na clínica odontológica, tanto nos procedimentos clínicos agendados como nos atendimentos de primeiros cuidados nas urgências, as emergências. Também verificou-se como eram feitos os procedimentos coletivos, como escovação supervisionada, evidenciação de placa bacteriana e bochechos fluorados, os quais eram agendados em grupo e realizados no 'escovódromo', uma ante-sala da clínica com uma grande pia, onde se escovavam os dentes, com cadeiras para os pacientes se sentarem para assistir às orientações, as quais eram feitas pelas THDs e tinham como foco a higiene bucal.

$\mathrm{Na}$ análise documental foi feita uma síntese de informações consideradas relevantes para o conhecimento da produção da assistência à saúde bucal realizada na UBS de estudo, tendo em vista uma complementação dos dados qualitativos, objeto central desta pesquisa.

Das informações selecionadas obtevese uma média dos três meses de produção, levando-se em conta somente a população adulta, excluindo os menores de 18 anos de acordo com o critério pré-estabelecido.

Eram realizados pela ESB em média os seguintes procedimentos: 66 procedimentos de $1^{a}$ Consulta Programática - é aquela em que o exame clínico odontológico do paciente é realizado com finalidade de diagnóstico e, necessariamente, elaboração de um plano preventivo- terapêutico (PPT), no âmbito de um programa de saúde ${ }^{9}$. Ela é anotada apenas uma vez ao ano. Foram realizados 57 tratamentos completados. O tratamento era considerado completado levando-se em conta os procedimentos no âmbito da atenção básica, não considerando a necessidade do usuário da referência ao serviço de atenção secundária ou terciária em saúde bucal. Quantos aos procedimentos coletivos foram 35. Compostos por 10 a 15 usuários representavam $20 \%$ da carga horária semanal do dentista. Os procedimentos coletivos eram realizados de diferentes formas pela equipe de saúde bucal. Ocorreram 88 atendimentos de urgência.

Em seguida, foi feita a análise das entrevistas. No total foram respondidos 25 questionários e conforme já relatado, por usuários adultos maiores de 18 anos, que tiveram algum atendimento odontológico nos últimos seis meses na UBS pesquisada.

\section{Características sóciodemográficas dos usuários}

As características sóciodemográficas da população de estudo incluiram as variáveis sexo, idade, estado civil, endereço, formação escolar, renda familiar e tempo de uso da Unidade Básica de Saúde estudada.

Com relação à variável sexo tivemos um maior número de usuários constituído pela população feminina. As idades que prevaleceram foram de 20 a 30 anos e de 40 a 60 anos. Quanto ao estado civil, não houve diferença entre solteiros e casados que se equiparam, havendo uma pequena parte formada por viúvos, separados ou divorciados.

A grande maioria residia no bairro Casa Verde Alta - SP, pois como preconiza o PSF, os usuários são atendidos por área, devendo morar numa determinada região. A variável tempo de utilização da UBS estudada mostrou que os usuários entrevistados já utilizavam o serviço há muito tempo, o que caracteriza um contingente de pessoas que tem grande possibilidade de conhecer realmente as qualidades e os 
problemas dessa UBS, o que tornou suas falas de importância ímpar.

Quanto à formação escolar tirando a categoria superior completo, constituída por uma parcela muito pequena, as outras categorias $\left(1^{\circ}\right.$ grau incompleto e completo e $2^{\circ}$ grau incompleto e completo) se equipararam. E finalmente, quanto à renda familiar mensal, a grande maioria foi dos que ganhavam até 2 salários mínimos.

\section{Análise das narrativas dos usuários:}

\section{Vínculo entre Usuário e Profissional de Saúde}

Uma das políticas implementadas no SUS é a Política Nacional de Humanização da Atenção e Gestão no Sistema Único de Saúde - PNH ou Humaniza SUS, que implica entre outros objetivos, em estabelecer vínculos entre usuário e trabalhador da saúde ${ }^{10}$.

No conteúdo de alguns depoimentos verificou-se a insatisfação de alguns usuários diante do fato de estar sempre trocando de dentista, apesar de passar sempre na mesma UBS:

- "eu já passei com 5 dentistas, Dr. A, Dr. B, Dra X, Dra Y e Dra Z. Os dentistas saem do postinho, eles vão para um lugar melhor, trabalhar em um lugar melhor, ai entra outro no lugar e a gente tem que ser atendido pelos dentistas que estão na hora”. (Usuário 5)

- "antes era a Dra X que me atendia, agora é outra, a Dra Y, não sei porque mudou, acho que a Dra X saiu." (Usuário 7)

- "eu venho aqui há muitos anos e os dentistas sempre mudam, já passei aqui com uns 6 ou7 dentistas." (Usuário 13)

- "o governo troca os dentistas sempre, não deveria trocar, pois quando a gente acostuma com um, eles vem e trocam" (Usuário 5)

- "esse é o segundo dentista que me atende, antes era o Dr. A, ai ele saiu e agora é o Dr. B. " (usuário 14)

- "passo com profissionais diferentes cada vez que venho." (Usuário 18).
Percebe-se, nas falas de alguns entrevistados, o desejo de manter o tratamento sempre com o mesmo profissional, reforçando o princípio de que só com o tempo se adquire confiança e se estabelece vínculo entre as pessoas. Uma relação de confiança e vínculo é fundamental para o bom andamento de um tratamento odontológico, por ser um tipo de assistência que enfrenta variadas situações que envolvem sentimentos de medo, de traumas, que algumas vezes impedem o usuário de se tratar.

Há uma associação entre o medo e a saúde bucal deficiente, que pode ter sido decorrente da menor freqüência de procura por tratamento odontológico pelos pacientes que tinham medo. Normalmente, os pacientes ansiosos esperam longos períodos para marcar uma consulta e não raramente a cancelam. Entre as principais causas que levavam o paciente a não buscar o dentista, estão o custo $(75,4 \%)$ e o medo $(36,9 \%)$. Dentre pessoas que não fizeram consultas odontológicas de rotina, $23 \%$ não o fizeram por medo".

Dessa forma, é necessário conseguir manter os dentistas por mais tempo no programa, sempre na mesma unidade, assim como descobrir o porquê dessa troca que descontenta os usuários e compromete o vínculo e consequentemente a humanização.

\section{Acesso aos serviços de saúde bucal}

Como já foi dito, a inserção da saúde bucal no PSF teve como uma das principais metas, ampliar o acesso da população à assistência odontológica no Brasil: "com o objetivo de reorganizar o modelo de atenção à saúde e ampliar o acesso às ações de saúde bucal, garantindo-se a atenção integral aos indivíduos e às famílias, Equipes de Saúde Bucal passaram a fazer parte da estratégia Saúde da Família" ${ }^{12}$.

O número de ESBs saltou de 4.261 para 17.349 no período de 2002 a 2008, estando presente em 4.857 dos 5.564 municípios brasileiros, representando um potencial de cobertura de mais de 91 milhões de habitantes, o que significou um aumento de $30 \%$ na cobertura ${ }^{13}$. 
Pode-se perceber que o PSF vem contribuindo para algumas mudanças, pois, por meio das falas dos usuários ficou claro que a 'acessibilidade', como porta de entrada ao serviço, tem aumentado. A grande maioria dos entrevistados relatou ser a primeira vez que foi atendida na rede pública para tratamento odontológico, sendo esta atenção em saúde bucal do PSF, a primeira oportunidade que tiveram de serem tratadas pelo SUS:

"Antes (do tratamento no PSF) eu passava no pronto-socorro público, mas não fazia tratamento, só arrancava dente." (Usuário12)

"Eu nunca tinha conseguido tratamento gratuito, antes eu tratava os dentes no sindicato dos gráficos, que meu pai era sócio, mas depois eu perdi o direito de ir lá e não tinha mais onde tratar, até eu conseguir aqui." (Usuário15)

"Eu só consegui ser atendido até hoje em pronto-socorro, para emergência, mas nunca fiz tratamento de dente, lá no pronto-socorro eles só arrancavam. (Usuário 25)

"Uma vez eu já tentei fazer tratamento de dente em outro posto aqui do bairro, mas lá é muita gente para ser atendida e não chamaram. Aqui não, logo chamam a gente e você é atendido." (Usuário 21)

"Eu sempre tive muito problema com meus dentes, principalmente na gravidez, mas nunca fiz tratamento, sempre sofri muito, só quando consegui aqui que melhorou, porque logo me marcaram para tratamento." (Usuário 19)

Esse aumento no acesso aos serviços de saúde bucal deve-se ao trabalho dos ACS, pois quase a totalidade dos entrevistados, quando perguntada sobre como foi que conseguiram atendimento, relatou que seu agendamento para o dentista foi feito a convite do ACS.

Porém, se por um lado aumentou o acesso, também há um maior número de pessoas para o atendimento, o que pode provocar um aumento da demanda aos serviços de atenção secundária e terciária em odontologia. Assim, maior acessibilidade exigirá do serviço maior organização para poder encaminhar os pacientes a esses serviços e garantir a integralidade das ações.

\section{A integralidade da atenção em saúde bucal. A Referência e Contra-referência}

O Ministério da Saúde determina que um dos princípios da inclusão da saúde bucal no PSF seja a integralidade. As ações de saúde bucal desenvolvidas nos serviços municipais ou regionais estão inseridas no primeiro nível de atenção denominado de atenção básica, porém os usuários devem ser encaminhados aos serviços de referência quando for requerida maior complexidade tecnológica para a resolução de situações ou de problemas identificados na atenção básica, garantindo-se assim a atenção integral aos indivíduos e às famílias.

Ainda que o acesso a serviços de saúde bucal esteja melhorando no Brasil, a atenção integral, preconizada pelo SUS, não têm sido alcançada na maioria dos municípios, havendo uma grande desproporção na oferta entre procedimentos odontológicos básicos e especializados $^{14 .}$

Em dezembro de 2000, por meio da portaria 144/GM, a saúde bucal começou a fazer parte do PSF. Foi regulamentada em março de 2001 , através da portaria $\mathrm{n}^{\circ} 267$, a qual tem no seu anexo 1 o Plano de Reorganização das Ações de Saúde Bucal na Atenção Básica, que estabelece, dentre outras coisas que a inclusão das ações de saúde bucal no PSF deverá expressar os princípios e diretrizes do SUS e apresentar a articulação da referência e contra-referência aos serviços de maior complexidade do Sistema de Saúde (grifo da autora) ${ }^{15}$.

Porém, surgiu em algumas falas dos entrevistados a questão da falta de serviços especializados em saúde bucal, no caso a falta da articulação de referência e contra-referência no programa estudado, expressando que tornava difícil ao usuário completar seu tratamento, comprometendo assim a integralidade da as- 
sistência, por falta dos serviços de atenção secundária:

"Eu estou com os dentes faltando e não tenho onde colocar uma prótese." (usuário 21).

De acordo com Frazão e Narvai13: "a partir de 2004, começaram a ser instalados em todos os estados brasileiros, com base na portaria $\mathrm{n}^{\circ} 1.570 / \mathrm{GM}$, os Centros de Especialidades Odontológicas (CEO), com o objetivo de ampliar e qualificar a oferta de serviços odontológicos especializados. Os CEO são unidades de referência para as unidades de saúde encarregadas da atenção odontológica básica".

Tais serviços de atenção secundária em saúde bucal funcionam na região em que foi realizada a pesquisa, no entanto esse funcionamento não é de conhecimento da população. Segundo o Ministério da Saúde ${ }^{10}$, "entre os resultados esperados com a $\mathrm{PNH}$ entende-se que todo usuário do SUS saberá que são os profissionais que cuidam da sua saúde e a rede de serviços que se responsabilizará por sua referência territorial (grifo da autora)".

No entanto, os sujeitos envolvidos na pesquisa não tinham nenhum ou tinham muito pouco conhecimento da existência dos CEOs e dos serviços de referência utilizados, assim como dos serviços de especialidades em odontologia e foi uma necessidade indicada quase que por unanimidade dos entrevistados quando perguntados sobre alguma sugestão que poderiam dar para melhorar $\mathrm{o}$ atendimento naquela UBS:

"Aqui poderia fazer prótese, eu preciso muito colocar uns dentes que faltam e não arrumo lugar para fazer" (Usuário 8).

"Ter mais especialistas aqui, eu acho que eles só fazem o básico, poderia ter outros especialistas, ou pelo menos lugares mais perto para poderem encaminhar" (Usuário 16).

E ainda: "Colocar mais dentistas que façam prótese, porque eu preciso de dentadura e não posso pagar, então, não tenho onde colocar." (Usuário 20)
A busca do usuário, por uma odontologia especializada ficou nítida nas falas dos sujeitos da pesquisa. É claro que está havendo um desencontro entre a busca do usuário e a tentativa do Sistema Único de Saúde em garantir a integralidade da assistência. Propõe-se assim, buscar uma solução, por meio da identificação e solução dos entraves que provocam esses desencontros, a fim de que os Centros de Especialidades Odontológicos possam realmente receber esses usuários que tanto anseiam pelo serviço.

Ao que parece, no presente estudo, a organização do serviço também não está conseguindo garantir a referência dos usuários aos CEOs e assim melhorar o acesso aos serviços de atenção secundária.

\section{A equidade no trabalho da equipe}

$\mathrm{Na}$ área da saúde pública, o conceito de equidade tem gerado amplos debates em diferentes países. No Brasil o tema é muito complexo devido a uma vigência de uma situação social marcada pela grande desigualdade. Segundo Pelicioni ${ }^{16}$, valores éticos como a equidade, a solidariedade e a justiça social devem ser usados a serviço de opções conscientes. Deste modo, o SUS representa uma política de inclusão social que tem a equidade como um de seus princípios.

O PSF, propondo-se a ser equânime, instalou-se na cidade priorizando as regiões periféricas de maior exclusão social. Houve portanto neste trabalho, o interesse em saber como está sendo aplicado na UBS estudada o princípio da equidade (termo que aqui se refere à oferta, acesso e utilização dos serviços) tentando verificar como o serviço tem organizado suas estratégias particulares no sentido de promover uma assistência que possibilite a realização igualitária das necessidades de seus usuários.

Segundo Zioni, Chiori e Almeida ${ }^{17}$, com respeito ao princípio da equidade, os serviços de saúde devem saber quais são as diferenças dos grupos da população e traba- 
lhar cada necessidade, oferecendo mais a quem mais precisa, diminuindo as desigualdades existentes. O SUS não pode oferecer o mesmo atendimento a todas as pessoas, da mesma forma e em todos os lugares. Se isto ocorrer, algumas pessoas vão ter o que não necessitam e outras não serão atendidas naquilo que necessitam, assim a idéia é tratar desigualmente os desiguais.

Algumas falas puderam levar à discussão de critérios utilizados para determinar as prioridades da assistência, pois, embora se proponha um atendimento igualitário, em determinadas situações, torna-se necessária uma hierarquização das prioridades, dando mais atenção a quem mais precisa. No entanto, isso parece ser sentido por alguns como injustiça e descaso para com sua dor:

"Eu falo dos meus problemas e eles marcam para um ano depois, é muito tempo, o problema não é resolvido rapidamente e se eu estou me queixando é porque preciso que resolvam logo, tem muita gente aqui. A demanda é muita para pouco profissional" (Usuário 15).

Esse usuário foi marcado para um ano depois, provavelmente porque a ESB assim entendeu como necessário devido a sua classificação de risco. No entanto, essa prioridade, determinada na organização do serviço, é sentida pelo paciente como injusta ou iníqua, pois não atendeu às suas necessidades.

Outro paciente sentiu-se injustiçado apesar de não padecer de nada muito grave, por não ter muita atenção:

"Eu acho que eles dão preferência para os que estão em estado mais grave, aqueles que não têm muita gravidade como eu, eles não dão muita atenção" (Usuário 13).

Terão esses usuários sido corretamente informados de que no PSF o serviço de saúde estabelece normas para a definição de prioridade no atendimento por meio da avaliação de risco dos agravos à saúde, a partir de critérios epidemiológicos da sua área de abrangência? Possivelmente, não!
Quanto à oferta de vagas para o atendimento, apareceu como insatisfação a questão das faltas às consultas marcadas, também sentida como injusta:

"Eu acho que eles deveriam ser menos rigorosos com a questão do comparecimento nas consultas, pois quando a gente não vai poder vir na consulta e vem remarcar, eles remarcam para depois de 6 meses e se você não pode vir e não consegue avisar, mesmo que for uma vez, já perde a vaga, eu trabalho fora o dia todo, tenho filha para cuidar, cuido da casa e deveria ter o direito de esquecer pelo menos uma vez a consulta" ( Usuário 14).

"Eu acho que eles poderiam ser mais compreensivo em caso de falta, as vezes a gente está marcado e tem aparelho quebrado e eles não nos atende, eles remarcam, e a gente entende, mas se a gente falta eles reclamam, fala que vai perder a vaga. Não acho que só nós que temos que entender o lado deles e não eles o nosso" (Usuário 4).

Também se verificou nas falas dos usuários que o tempo determinado pela equipe para o retorno à consulta depois de encerrado o tratamento era visto como injusto:

"gostaria que o retorno nosso fosse de 6 em 6 meses, hoje em dia eles marcam para a gente retornar depois de um ano, eu acho pouco vir ao dentista de ano em ano, eu sempre ouvi falar que era pra vir em dentista de $6 \mathrm{em}$ 6 meses" (Usuário 25).

"eu acho que o nosso retorno depois que termina o tratamento deveria ser de $6 \mathrm{em} 6$ meses e não de ano em ano como é agora" (Usuário 16)

As diretrizes para a atenção em saúde bucal da Secretaria Municipal da cidade de São Paulo possuem uma programação de retornos, conforme a classificação de risco. O período para o retorno varia conforme o tipo do agravo e o grupo de risco em que se encontra o paciente $^{18}$.

Porém parece que os profissionais da equipe de saúde bucal da Unidade Básica de 
Saúde estudada não estão totalmente conscientes dessas diretrizes e não informaram os pacientes desses critérios e os motivos pelos quais alguns tinham que retornar com mais freqüência às consultas, outros menos. Foi essa falta de informações que acabou por provocar nos usuários a sensação de injustiça.

Sendo essa pesquisa um estudo de caso, evidentemente não permite generalizações e tem uma limitação temporal e geográfica. Assim sendo, seus dados são restritos à UBS estudada.

\section{CONSIDERAÇÕES FINAIS}

A recente inserção da saúde bucal no PSF representou um avanço em saúde bucal coletiva. No município de São Paulo esta inserção é dinâmica e está em constante processo de evolução.

A análise e discussão dos resultados permitiram verificar que, na UBS estudada o PSF está contribuindo para um avanço da saúde bucal coletiva no SUS. Porém as percepções dos usuários mostraram algumas subjetividades relevantes que, se consideradas, podem trazer uma maior aproximação do serviço com a comunidade, na realização de mudanças efetivas no modelo de atenção, superando problemas e assumindo compromissos pautados para responder às necessidades e expectativas dos indivíduos e famílias da região.

Ficou claro que o PSF não está conseguindo proporcionar uma relação positiva entre usuário e profissional de saúde onde se estabeleça vínculo, pois de acordo com as falas já apresentadas, eles acabaram sendo atendidos por vários dentistas no decorrer do tratamento.

Percebeu-se o desejo dos entrevistados de manter o tratamento sempre com o mesmo profissional, partindo do princípio de que só com o tempo se adquire confiança e vínculo com o dentista.

Quanto ao acesso ao serviço de saúde bucal, o PSF está conseguindo avançar, pois a maioria dos entrevistados relatou que o trata- mento aí realizado foi o primeiro de suas vidas e mesmo com relação ao acesso dos usuários ao serviço de saúde como porta de entrada do sistema, o acesso melhorou com o Programa.

Entretanto, esse mesmo aumento de acesso aos serviços de saúde bucal gerou maior demanda por serviços especializados e os usuários que deles necessitavam não estavam sendo a eles encaminhados.

A falta de serviços de especialidades odontológicas foi a queixa principal dos entrevistados. Eles não conheciam nenhum serviço para atendimento especializado em odontologia e não sabiam que no bairro havia o CEO.

O problema da referência aos serviços de atenção secundária em odontologia destacou-se pela ausência de serviços especializados como prótese e endodontia.

Percebeu-se que as práticas de saúde bucal da UBS não estavam conseguindo resolver a maior parte dos problemas de saúde da sua população atendida, comprometendo a integralidade do atendimento.

Quanto à equidade, na percepção do usuário algumas características do atendimento eram iníquas, confirmando a certeza de que pessoas com diferentes necessidades não podem estar recebendo o mesmo padrão de serviços de saúde bucal.

A sensação de injustiça relatada pelos usuários referia-se a algumas normas no atendimento: o tempo de retorno à consulta depois de terminado o tratamento que eles julgaram muito longo um ano na maioria dos casos; a questão da perda da vaga devido às faltas foi ainda considerada por alguns, como total incompreensão e injustiça. A falta de orientação e informação acabava provocando nos usuários uma sensação de injustiça.

Neste sentido, este estudo conseguiu mostrar que a inserção da saúde bucal no PSF ainda apresenta algumas questões relevantes que dificultam a concretização de um modelo de atenção eficaz caracterizado pela humanização do atendimento, a integralidade em saúde bucal, a equidade e o acesso. 


\section{REFERÊNCIAS}

1. Pinto VG. Epidemiologia das Doenças Bucais no Brasil. In: Kriger L, organizador. Promoção de Saúde Bucal: paradgima, ciência, humanização. $3^{\circ}$ ed. São Paulo; Artes Médicas; 2003. p.1-24

2. IBGE - Instituto Brasileiro de Geografia e Estatística; Anuário Estatístico do Brasil, 20O3. Rio de Janeiro, 2004.

3. Moysés SJ, Nascimento AC, Gabardo MCL, Dittrich R. Apontamentos para estudos e debates sobre a Estratégia Saúde da Família. In: Moysés ST, Kriger L, Moysés SJ, coordenadores. Saúde Bucal das Famílias, trabalhando com evidências. São Paulo: Artes Médicas, 2008, p.47-64.

4. Turato ER, Ricas J, Fontanella BJB. Amostragem por Saturação em Pesquisas Qualitativas de Saúde: contribuições teóricas. Cad de Saúde Pública, Rio de Janeiro, 24(1), jan. 2008.

5. Yin RK. Estudos de caso: planejamento e métodos. $3{ }^{\text {a }}$ ed., Porto Alegre: Bookmam, 2005. 212p

6. Zioni F, Souza DV: "Pesquisa social: métodos aplicados ao saneamento". In: Philippi, AJ ( Org.). Saneamento, Saúde e Ambiente - fundamentos para um desenvolvimento sustentável. $1^{\text {a }}$ Ed., Barueri, SP, Ed. Manole, 2005, p. 599-622.

7. Minayo MCS. O desafio do conhecimento: Pesquisa qualitativa em saúde. 8.ed. São Paulo/Rio de Janeiro: Hucitec, 1994.

8. Bardin L. Análise de conteúdo. Lisboa: Edições 70, 2000.

9. Brasil. Ministério da Saúde. Agência Nacional de Vigilância Sanitária. Serviços Odontológicos: prevenção e controle de riscos. Brasília, DF, 2006.

10. Brasil. Ministério da Saúde. Política Nacional de Humanização - PNH. Brasília, DF, 2004.

11. Rosa AL, Ferreira CM. Ansiedade Odontológica: nível de ansiedade, prevalência e comportamento dos indivíduos ansiosos. Revista Brasileira de Odontologia, São Paulo, 1997.

12. Brasil, Ministério da Saúde. Secretaria de Atenção Básica. Departamento de Atenção Básica. Saúde Bucal. Brasília, DF, 2006 (Cadernos de Atenção Básica n.17).

13. Frazão P, Narvai PC. Saúde bucal no Sistema Único de Saúde : 20 anos de lutas por uma política pública. Saúde em Debate, Rio de Janeiro, v.33 n.81, jan./abr. 2009.

14. Figueiredo N, Goes PSA. Construção da atenção secundária em saúde bucal: um estudo sobre os Centros de Especialidades Odontológicas em Pernambuco, Brasil. Cad de Saúde Pública. Rio de Janeiro, v.25, n.2, p.259-267, fevereiro de 2009.

15. Brasil. Ministério da Saúde. Departamento de Atenção Básica. Guia Prático do Programa de Saúde da Família. Brasília, DF, 2001.

16. Pelicioni MCF. Educação em Saúde e Educação Ambiental: estratégias de construção da escola promotora da saúde [tese de livre-docência]. São Paulo: Faculdade de Saúde Pública da USP, 2000.

17. Zioni F, Chioro A; Almeida, ES. Políticas Públicas e Organização do Sistema de Saúde: Antecedentes, Reforma Sanitária e o SUS. In: Westphal MF, Almeida ES, organizadores. Gestão de Serviços de Saúde. $1^{a}$ ed. São Paulo; Edusp; 2001.

18. São Paulo (cidade). Secretaria Municipal de Saúde. Coordenação da Atenção Básica. Área Técnica de saúde Bucal. Diretrizes para a Atenção em Saúde Bucal: crescendo e vivendo com saúde bucal. São Paulo, 2009. 